\title{
FACTORES ORGANIZACIONALES QUE INFLUYEN EN LA GENERACIÓN DE VINCULACIÓN ENTRE EMPRESAS Y UNIVERSIDADES
}

\author{
Ramón Antonio Valle Morales* \\ http://orcid.org/0000-0002-9158-0420 \\ Iván Tapia Moreno** \\ http://orcid.org/0000-0001-9280-5321
}

RECIBIDO: Abril 2019 / ACEPTADO: Septiembre 2019 / PUBLICADO: Enero 2020

\begin{abstract}
Como citar: Valle Morales, Ramón Antonio; Tapia Moreno, Iván. (2020). Factores organizacionales que influyen en la generación de vinculación entre empresas y universidades. Telos: revista de Estudios Interdisciplinarios en Ciencias Sociales, 22 (1), Venezuela. (Pp.45-61).

DOI: www.doi.org/10.36390/telos221.04
\end{abstract}

\section{RESUMEN}

El presente artículo tuvo como objetivo identificar los factores organizacionales que influyen en la generación de vinculación entre empresas y universidades. Se asume una metodología mixta para la recolección y el análisis de datos cuantitativos y cualitativos en el método que forma parte de la investigación, en donde la muestra fue por conveniencia y estuvo constituida por diez (10) directivos de empresas a nivel estatal. Se utilizó como técnica la entrevista semi estructurada, fundamentada en un instrumento tipo guía de entrevista dirigido a todos los directivos de las empresas. Se revisan documentos especializados con el fin de realizar una disertación teórica inicial, tales como Pérez (2016), Alcántar y Arcos (2004), Chiang, et al (2008), Saavedra (2009), Strauss y Corbin (1990), entre otros. Los resultados indican que para la generación de vinculación entre las empresas y universidades es de gran relevancia que tengan conocimiento de los factores organizacionales que permitan la generación de proyectos de vinculación. Así mismo, con base a los resultados obtenidos se desarrolló propuesta de modelo basado en teoría fundamentada que incluye los factores internos, trabajo colaborativo, desarrollo de talento humano, área de vinculación y tipo de vinculación como factores organizacionales identificados durante el proceso de teoría fundamentada. El artículo propone un punto de partida para realizar investigaciones futuras que permitan corroborar su utilidad y puesta a punto. La necesidad de trabajo futuro es clara, sobre todo para dar seguimiento a los hallazgos encontrados durante el desarrollo de la investigación.

Palabras clave: Vinculación, factores organizacionales, universidad, empresa, teoría fundamentada.

\footnotetext{
* Estudiante Maestría en Tecnologías de Información para los Negocios en el Instituto Tecnológico de Sonora. Ciudad Obregón, Sonora, México. Correo electrónico: ramon.valle@potros.itson.edu.mx

${ }^{* * *}$ Profesor Investigador del Instituto Tecnológico de Sonora. Ciudad Obregón, Sonora, México. Correo electrónico: itapia@itson.edu.mx
} 
Factores organizacionales que influyen en la generación de vinculación entre empresas y universidades

\title{
Organizational factors that influence the generation of links between companies and universities
}

\begin{abstract}
The objective of this article was to identify the organizational factors that influence the generation of links between companies and universities. It assumes a mixed methodology for the collection and analysis of quantitative and qualitative data in the method that is part of the research, where the sample was for convenience and was composed of ten (10) managers of companies at the state level. The semi-structured interview was used as a technique, based on an interview guidetype instrument aimed at all company managers. Specialized documents are reviewed in order to carry out an initial theoretical dissertation, such as Pérez (2016), Alcántar and Arcos (2004), Chiang, M. et al (2008), Saavedra (2009), Strauss and Corbin (1990), among others. The results indicate that for the generation of linkage between companies and universities it is of great relevance that they have knowledge of the organizational factors that allow the generation of linking projects. Likewise, based on the results obtained, a model proposal was developed based on grounded theory that includes internal factors, collaborative work, human talent development, bonding area and type of linkage as organizational factors identified during the theory-based process. The article proposes a starting point to carry out future research to corroborate its usefulness and fine-tuning. The need for future work is clear, especially to follow up on the findings found during the development of the research.
\end{abstract}

Key words: Linkage, organizational factors, university, business, grounded theory.

\section{Introducción}

La transferencia de conocimiento se ha vuelto una estrategia de competitividad esencial para el crecimiento de las empresas, donde el principal ente que genera diversas innovaciones del conocimiento son las instituciones de educación superior (IES). Razón por la cual, el conocimiento debe de ser aplicado a la realidad de la industria para lograr solucionar los problemas presentados en ella, permitiendo que logren vincularse con el sector empresarial (Pérez, 2016).

Alcántar y Arcos (2004), definen vinculación como la actividad que permite sostener una relación entre las universidades y las empresas. Este término se ha puesto de moda en la última década, y forma parte de la jerga de los especialistas en educación superior para aludir con un nuevo matiz a la misma dimensión que denotan los términos difusión y extensión. No obstante, el uso frecuente y restringido del término lo limita a las relaciones de las instituciones educativas con industrias u otras empresas del llamado sector productivo de la sociedad.

Por lo anterior, tanto en las empresas y universidades surge la necesidad de identificar cuáles son los factores organizacionales con los que cuentan y que influyen para generar la vinculación. Chiang, et al. (2008), menciona que la misión es la razón de ser de una organización, y su visión es el objetivo a alcanzar, pero también es importante para la productividad su estructura y el conjunto de personas con responsabilidades específicas, que actúan juntas para el logro de los objetivos trazados por dicha organización.

Es por esto que las empresas están cada vez más conscientes de la importancia del talento humano para la mejora de resultados, a través de la búsqueda constante de circunstancias 
idóneas que les permitan ser capaces de innovar y poner en práctica ideas brillantes y enriquecedoras para dar esa ventaja competitiva que toda organización precisa para triunfar. En tal sentido, el objetivo de este estudio es analizar los factores organizacionales que influyen en la generación de la vinculación entre las empresas y universidades.

Por lo tanto, para el cumplimiento del objetivo este documento consta de cuatro apartados. En el primero se presentan los trabajos relacionados en donde se revisan contribuciones sobre el tema con el fin de identificar la importancia que genera para las empresas y universidades trabajar en conjunto para el logro de sus objetivos, es decir, interactuar entre ellos a través de los factores organizacionales que influyen en la generación de la vinculación.

El método de investigación es presentado en el siguiente apartado. Para cumplir con el objetivo de la investigación se parte de una perspectiva de análisis centrada en los informantes y sus percepciones a través de una entrevista semi-estructurada. Así mismo, se presenta en este apartado la estrategia metodológica, las técnicas e instrumentos utilizados durante la investigación para obtener la información, así como de los procedimientos empleados para su procesamiento.

En el tercer apartado se presentan los resultados de la investigación, donde se muestran las categorías principales identificadas a través de la codificación abierta, así como sus propiedades y dimensiones, así también se muestra modelo propuesto de teoría fundamentada para responder la interrogante de investigación, al final del artículo, en el cuarto apartado, se realiza un breve recuento de las principales conclusiones del estudio.

\section{Trabajos relacionados}

Para lograr identificar la importancia que genera en las empresas y universidades trabajar vinculados y lograr con ello el alcance de sus objetivos, se presentan las ideas de un conjunto de artículos analizados.

Pérez (2016), refiere que tanto la universidad como la empresa, deben considerar la importancia de colaborar de manera conjunta, relacionando sus objetivos y teniendo la idea de que necesitan interactuar entre ellos para obtener mayores beneficios y ser más competitivos. Es por esto que las empresas y universidades deben de contar con el recurso humano capacitado y con las habilidades necesarias para lograr sus objetivos, además de la infraestructura para el desarrollo de proyectos de investigación tal como indica Martínez (1999), la transferencia del conocimiento es identificable en diferentes formas, la primera es la formación de recursos humanos, esto va por parte de las universidades con la capacitación de estudiantes y académicos dotándolos de herramientas y habilidades, la segunda la conforman los recursos tecnológicos o también conocido como infraestructura para desarrollar proyectos de investigación y por último los recursos para la investigación y desarrollo los cuales pueden presentarse por medio de beneficios económicos para el investigador y así alentar a que estas prácticas se multipliquen con el paso del tiempo.

Por otra parte, Chiang et al. (2008), indica que el desafío de la universidad actual es refrendar su compromiso con la sociedad, preparando los recursos humanos que la misma necesita, para esto es necesario cerrar la brecha entre lo que se enseña en las universidades y lo que demanda el mercado laboral, estableciendo un constante monitoreo de la evolución de las empresas y ajustando constantemente la currícula de estudios como una respuesta a esas necesidades. Cuando las instituciones de educación superior hayan demostrado entonces que son capaces 
Factores organizacionales que influyen en la generación de vinculación entre empresas y universidades

de generar el capital humano idóneo y capaz de enfrentar la realidad, las empresas tendrán menos desconfianza y habrá mayor probabilidad de establecer la vinculación vía proyectos empresariales (Saavedra, 2009).

Así mismo, Kotler y Armstrong (2001), refieren que el diagnóstico organizacional parte de un análisis completo de la situación de la empresa, éste incluye la desagregación de los elementos externos a la organización, así como la consideración de aspectos internos; esto exige la realización de un análisis del ambiente interno de las empresas, identificación de factores, variables, actores o elementos existentes que de manera directa e indirecta afectan la vinculación de las empresas con las universidades.

En el contexto de ambientes altamente turbulentos la alta gerencia puede facilitar la formación de equipos de trabajo proveyendo seguridad psicológica, empoderamiento, motivación y cultivar capacidades de solución de problemas (Carbonell y Rodríguez-Escudero, 2009). La búsqueda de nuevas técnicas y su aplicación es altamente sensible a la cantidad y calidad de los recursos humanos, infraestructura de medición, prueba y experimentación (Lara, 2001).

Por lo antes mencionado, se considera la siguiente interrogante: ¿Cuáles son los factores organizacionales que influyen en la generación de la vinculación entre las empresas y universidades?

\section{Método de investigación}

Para responder a la interrogante de la investigación se utilizó una metodología mixta, en la cual se realizó un estudio cualitativo utilizando teoría fundamentada basada en una entrevista semi estructurada. Strauss y Corbin (1990), refieren que la teoría fundamentada (Grounded Theory) es un método de investigación en el que la teoría emerge desde los datos. Es una metodología que tiene por objeto la identificación de procesos sociales básicos (PSBs), como punto central de la teoría. A través de esta metodología podemos descubrir aquellos aspectos que son relevantes de una determinada área de estudio.

La teoría fundamentada utiliza una serie de procedimientos que, a través de la inducción, genera una teoría explicativa de un determinado fenómeno estudiado. En este sentido, los conceptos y las relaciones entre los datos son producidos y examinados continuamente hasta la finalización del estudio. Strauss y Corbin (1990), aseguran que si la metodología se utiliza adecuadamente reúne todos los criterios para ser considerada rigurosa como investigación científica.

Así mismo, para el desarrollo de la investigación se realizó investigación cuantitativa, como refiere Sarduy (2007), la investigación cuantitativa estudia la asociación o relación entre las variables que han sido cuantificadas, lo que ayuda aún más en la interpretación de los resultados.

Por lo cual, se realizó un estudio de investigación cuantitativa con el objetivo de recoger, procesar y analizar datos cuantitativos o numéricos sobre variables previamente determinadas. Lo anterior hace darle una connotación que va más allá de un mero listado de datos organizados como resultado; pues estos datos que se muestran en el informe final, están en total consonancia con las variables que se declararon desde el principio y los resultados obtenidos van a brindar una realidad específica a la que estos están sujetos.

\section{Instrumento}

Existe una gran diversidad de enfoques para identificar los factores organizacionales que influyen en la generación de la vinculación entre las empresas y universidades. Por lo anterior, 
se optó por elaborar una entrevista semi-estructurada (Ver anexo 1), la cual fue validada por expertos, mediante técnica de Likert, se revisaron 10 factores con 10 ítems cada uno, quedando finalmente 5 factores que en esta investigación se utilizaron con un total de 23 ítems. La tabla 1 muestra el nombre de las secciones y el número de preguntas que comprende cada sección de la entrevista elaborada.

La entrevista fue elaborada de manera semi estructurada, ya que como mencionan Díaz et al. (2013), este tipo de entrevistas presentan un mayor grado de flexibilidad que las entrevistas estructuradas, porque parten de una guía de preguntas planeadas que se pueden ir ajustando a los entrevistados.

Tabla 1. Descripción de la estructura de entrevista.

\begin{tabular}{|l|c|}
\hline \multicolumn{1}{|c|}{ Secciones } & Preguntas \\
\hline $\begin{array}{l}\text { Organización Interna de la empresa para generar la vinculación con las } \\
\text { universidades. }\end{array}$ & 7 \\
\hline Trabajo colaborativo para generar la vinculación con universidades. & 5 \\
\hline Desarrollo de talento humano en la empresa & 5 \\
\hline $\begin{array}{l}\text { Estrategias para disminuir la deserción del talento humano en las } \\
\text { empresas }\end{array}$ & 2 \\
\hline Participación de la empresa en proyectos de vinculación con universidades & 4 \\
\hline
\end{tabular}

Fuente: Elaboración propia.

\section{Muestra}

La investigación se llevó a cabo en Ciudad Obregón, Sonora, México. La entrevista fue aplicada a 10 directores de empresas dedicadas a la generación de soluciones de negocio a sus clientes a través de la asesoría integral de servicios de software y automatización de procesos, esta muestra se considera por conveniencia porque fue debido a la disponibilidad, noviembre 2018. El $80 \%$ de la muestra son personas del sexo masculino y el $20 \%$ restante son de sexo femenino. El perfil de los entrevistados considera personas que actualmente laboran como directores de empresas desarrolladoras de software.

Para la identificación y el reclutamiento de la muestra se seleccionó a 10 personas en total que trabajaran en distintas empresas de la región con el objetivo de lograr obtener un panorama más amplio. Una vez identificados, se les invitó de manera personal y correo electrónico a participar en el estudio. De los 10 entrevistados invitados, 10 aceptaron ser parte del estudio. Es importante hacer mención que todos los entrevistados participaron de forma voluntaria sin obtener algún tipo de compensación por su participación.

\section{Procedimiento}

El estudio fue realizado a través de los siguientes pasos. Una vez que los participantes aceptaran ser parte del estudio se les introdujo de forma general al tema y objetivo de la investigación. Posteriormente, cada participante fue entrevistado de forma individual en sesiones con una duración promedio de 37 minutos, en donde, cada sesión fue grabada con el fin de realizar el análisis con teoría fundamentada. 
Factores organizacionales que influyen en la generación de vinculación entre empresas y universidades

Todas las grabaciones de las entrevistas semi-estructuradas fueron transcritas. Después, a fin de obtener un entendimiento del fenómeno, se siguió la metodología de teoría fundamentada, para seguir un procedimiento estructurado y bien definido de adquisición de conocimiento, lo cual, se desarrolló de manera manual haciendo uso de tablas en Microsoft Excel para la integración de la información por categorías y dimensiones identificadas para cada categoría a través de la aplicación de las entrevistas, y posteriormente se realizó propuesta de modelo basado en teoría fundamentada.

Las diez entrevistas fueron contestadas en su totalidad, lo que llevó un porcentaje de $100 \%$ cuestionarios utilizados. Se capturó y procesó la información utilizando el paquete estadístico SPSS (Statistical Package for Social Sciences) versión 15 para Windows.

\section{Resultados \\ Cualitativos}

A pesar de que las empresas parecen conocer los factores generadores de vinculación con las universidades, pocas son las que los conocen formalmente, por ejemplo, trabajar de manera colaborativa y desarrollar el talento humano. Si se desea que las empresas tengan conocimiento de estos factores, deben de empezar por identificar cuáles son los factores organizacionales con los que cuentan para trabajar en conjunto con las universidades $y$, una vez identificados, puedan relacionar sus objetivos para obtener con ello mayores beneficios a través de la vinculación.

Por lo anterior, se realizó el procedimiento codificación abierta de la teoría fundamentada, en donde se identificaron cinco categorías principales: factores internos, trabajo colaborativo, desarrollo de talento humano, área de vinculación y tipo de vinculación. En la tabla 2 se describe cada categoría identificada. Así mismo, la tabla 3 muestra las propiedad y dimensiones para cada categoría identificada en el desarrollo de la codificación abierta.

\section{Tabla 2. Descripción de categorías identificadas.}

\begin{tabular}{|l|l|}
\hline \multicolumn{1}{|c|}{ Categoría } & \multicolumn{1}{c|}{ Descripción } \\
\hline Factores & $\begin{array}{l}\text { Se refiere a los factores internos con los que cuentan las empresas para llevar a } \\
\text { internos } \\
\text { labo la vinculación con las universidades, y estos factores incluyen, la estrategia, } \\
\text { factores primordiales para el desarrollo de proyectos de vinculación. }\end{array}$ \\
\hline $\begin{array}{l}\text { Trabajo } \\
\text { colaborativo }\end{array}$ & $\begin{array}{l}\text { Se refiere a las actividades profesionales en las que existe algún tipo de } \\
\text { colaboración entre personas, departamentos e incluso empresas creadas para } \\
\text { alcanzar un objetivo común. }\end{array}$ \\
\hline $\begin{array}{l}\text { Desarrollo de } \\
\text { Talento Humano }\end{array}$ & $\begin{array}{l}\text { Se refiere al conjunto de procesos de recursos humanos integrados y diseñados } \\
\text { para atraer, desarrollar, motivar y retener a los colaboradores de la empresa. Cabe } \\
\text { mencionar que esta categoría considera exclusivamente a las estrategias de } \\
\text { desarrollo del talento humano, así como a los beneficios que ofrecen las empresas } \\
\text { a sus colaboradores. }\end{array}$ \\
\hline $\begin{array}{l}\text { Área de } \\
\text { vinculación }\end{array}$ & $\begin{array}{l}\text { Se refiere al vínculo físico entre las empresas y la comunidad universitaria, para la } \\
\text { gestión, desarrollo y seguimiento de proyectos, mediante contratos o convenios de } \\
\text { colaboración. Así también se refiere al conocimiento de ideas y beneficios por parte } \\
\text { del responsable del área para que pueda ser un motivador de la vinculación entre } \\
\text { los involucrados. }\end{array}$ \\
\hline $\begin{array}{l}\text { Tipo de } \\
\text { vinculación }\end{array}$ & $\begin{array}{l}\text { Incorpora todo lo relacionado con aspectos de los procesos, involucrados, políticas } \\
\text { y herramientas de seguimiento para su desarrollo. }\end{array}$ \\
\hline
\end{tabular}


Fuente: Elaboración propia.

Tabla 3. Propiedades y Dimensiones para cada categoría identificada.

\begin{tabular}{|c|c|c|}
\hline Categoría & Propiedades & Dimensión \\
\hline Factores Internos & Características & $\begin{array}{l}\text { Apertura completa } \\
\text { Mayor tiempo para proyectos } \\
\text { Recursos económicos } \\
\text { Costos a absorber } \\
\text { Infraestructura } \\
\text { Hardware para realizar el } \\
\text { trabajo } \\
\text { Capacidad de inversión } \\
\text { Capacidad de tiempo } \\
\text { Espacio en las universidades }\end{array}$ \\
\hline Trabajo colaborativo & $\begin{array}{r}\text { Barreras } \\
\text { Beneficios } \\
\text { Necesidades }\end{array}$ & $\begin{array}{l}\text { Compromiso } \\
\text { Tiempo } \\
\text { Valor del proyecto } \\
\text { Área específica de } \\
\text { vinculación } \\
\text { Identificar áreas de } \\
\text { oportunidad } \\
\text { Innovar } \\
\text { Desarrollo empresarial } \\
\text { Penetrar en otros mercados } \\
\text { Mejorar la calidad } \\
\text { Confiabilidad } \\
\text { Asertividad en selección de } \\
\text { talento } \\
\text { Poder de negociación } \\
\text { Responsable de vinculación }\end{array}$ \\
\hline
\end{tabular}


Factores organizacionales que influyen en la generación de vinculación entre empresas y universidades

\begin{tabular}{|c|c|c|}
\hline $\begin{array}{l}\text { Desarrollo de Talento } \\
\text { Humano }\end{array}$ & $\begin{array}{l}\text { Estrategias } \\
\text { Retención } \\
\text { Beneficios }\end{array}$ & $\begin{array}{l}\text { Empoderamiento } \\
\text { Decisiones del proyecto } \\
\text { Libertad de correr riesgos } \\
\text { Prestaciones económicas } \\
\text { Trabajo colaborativo } \\
\text { Flexibilidad de horario } \\
\text { Espacio de trabajo } \\
\text { Escuchar sus ideas } \\
\text { Estabilidad de la empresa } \\
\text { Esquema de remuneración } \\
\text { Sueldo competitivo } \\
\text { Plan de carrera } \\
\text { Creación de comunidad } \\
\text { Involucra proyectos reales } \\
\text { Entrenamiento }\end{array}$ \\
\hline Área de vinculación & $\begin{array}{r}\text { Existencia } \\
\text { Responsable }\end{array}$ & $\begin{array}{l}\text { No -Sí } \\
\text { No -Sí } \\
\text { Conocimiento de las ideas } \\
\text { Logre identificar beneficios } \\
\text { Líder } \\
\text { Motivador } \\
\text { Vínculo universidad y } \\
\text { empresa }\end{array}$ \\
\hline Tipo de vinculación & $\begin{array}{r}\text { Tipo } \\
\text { Razones } \\
\text { Involucrados } \\
\text { Seguimiento }\end{array}$ & $\begin{array}{l}\text { Prácticas profesionales } \\
\text { Investigación nivel ciencia } \\
\text { Investigación nivel tesis } \\
\text { Ofrecer talento local } \\
\text { Trabajo en conjunto } \\
\text { Universidades } \\
\text { Sistemas } \\
\text { Software } \\
\text { Mecatrónica } \\
\text { No - Sí } \\
\text { Semanal - Mensual } \\
\text { Calendario de universidad }\end{array}$ \\
\hline
\end{tabular}

Fuente: Elaboración propia

Como resultado del proceso de teoría fundamentada, se diseñó modelo que trata de responder y explicar la interrogante de la investigación. La figura 1 muestra el modelo basado en teoría fundamentada propuesto que explica los factores organizacionales que influyen en la generación de la vinculación entre empresas y universidades. 
Figura 1. Propuesta de modelo basado en teoría fundamentada.

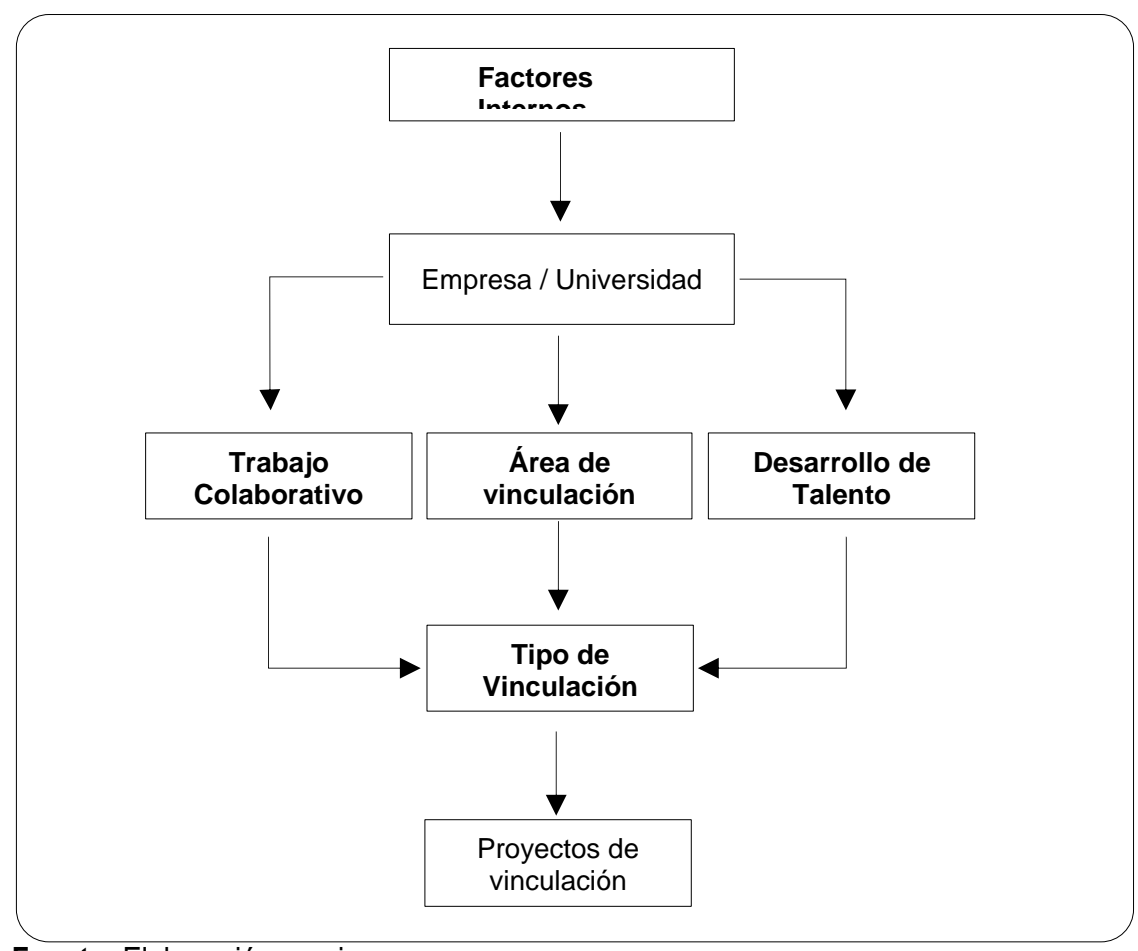

Fuente: Elaboración propia

Como se muestra en la figura 1 los factores organizaciones identificados en la elaboración de teoría fundamentada comprenden los factores internos de la empresa y universidad, es decir, la infraestructura, capacidad de inversión, hardware para realizar los proyectos y recursos económicos. Así también se identificó el factor de trabajo colaborativo el cual permitiría a las universidades identificar áreas de oportunidad para desarrollar la vinculación a través de proyectos de innovación, así como lograr una mejora en la penetración de otros mercados y su desarrollo empresarial.

Además, se identificó que el área de vinculación es un factor que influye en la generación de la vinculación entre las empresas y universidades, es decir, que en las empresas y universidades exista esta área, así como el responsable de área que tenga el conocimiento de los idiomas que se hablan en las empresas y universidades, de tal manera que permita identificar los requerimientos tanto de las empresas y universidades para generar ideas que les permitan obtener beneficios al desarrollar proyectos a través de la vinculación. Velázquez (2012), refiere importante señalar, que de acuerdo a la tendencia conocida como la "La era del conocimiento", las empresas deben de buscar una vinculación a fondo con las universidades, al ser ellas, 
Factores organizacionales que influyen en la generación de vinculación entre empresas y universidades

quienes generan y preparan a los nuevos talentos, los cuales, deberán estar alineados con las demandas y capacidades que requieren las empresas para ser competitivas.

Así mismo, actualmente, una empresa competitiva se caracteriza cada vez menos por su dependencia a la dotación de insumos físicos, y cada vez más, por su habilidad para explotar activos intangibles, tales como sus propias capacidades empresariales, es decir, su capital intelectual. "La capacidad de transformación que pueda exhibir una empresa para transitar de la explotación de insumos físicos al manejo eficiente del conocimiento, determina su verdadero grado de competitividad" (Bagella y Becchetti, 2000).

Así mismo, se identificó como factor organizacional el desarrollo de talento humano como influencia en la generación de la vinculación entre las empresas y universidades, en donde los entrevistados mencionan que las estrategias que llevan a cabo son el trabajo colaborativo, flexibilidad de horario, empoderamiento por parte de los colaboradores en los proyectos que desarrollan, así como prestaciones económicas, capacitación y entrenamiento para los colaboradores de la empresa. Porter (1998), señala en su Diamante de la Competitividad, que "una educación de calidad hace competitivas a las industrias de soporte", bajo esta óptica, para que las empresas mexicanas sean competitivas a nivel mundial, es necesario desarrollar no sólo la infraestructura necesaria, sino una sector con industrias soporte, que este alimentando a los otros sectores con los insumos necesarios, uno de ellos es el capital humano, por ello las empresas deben de estar fuertemente ligadas a las universidades, para obtener recursos humanos calificados con las competencias que requieren las organizaciones para generar innovaciones y solucionar problemas.

Finalmente, se identificó que el tipo de vinculación es otro de los factores organizacionales que influyen en la generación de la vinculación entre las empresas y universidades, en donde es de gran importancia identificar el tipo de vinculación a desarrollar, así como tener conocimiento de los procesos, involucrados, políticas y herramientas de seguimiento para el desarrollo de proyectos de vinculación.

\section{Cuantitativos}

Por otra parte, la entrevista con 23 ítems fue aplicado a 10 directivos de empresas de Ciudad Obregón, Sonora. La muestra se considera por conveniencia porque fue debido a la disponibilidad, noviembre 2018. Se capturó y procesó la información utilizando SPSS versión 15.

A continuación, en la Tabla 4 se presentan los datos obtenidos por factor organizacional de acuerdo a lo mencionado por los entrevistados. En donde, cada uno de los valores representan el total de veces que el entrevistado hizo mención de dimensiones relacionadas con cada uno de los factores. Por mencionar, el IN1 (Informante 1) hizo mención de 8 dimensiones relacionadas con el factor organizacional AV (Área de vinculación).

Tabla 4. Datos de entrevistas.

\begin{tabular}{|c|r|r|r|r|r|}
\hline INF & AV & FI & TH & TV & TC \\
\hline IN1 & 8 & 8 & 8 & 2 & 6 \\
\hline IN2 & 7 & 10 & 9 & 3 & 8 \\
\hline IN3 & 7 & 9 & 8 & 8 & 8 \\
\hline IN4 & 8 & 8 & 8 & 7 & 8 \\
\hline
\end{tabular}




\begin{tabular}{|c|r|r|r|r|r|} 
IN5 & 8 & 7 & 7 & 6 & 7 \\
\hline IN6 & 8 & 7 & 9 & 7 & 8 \\
\hline IN7 & 7 & 6 & 6 & 6 & 9 \\
\hline IN8 & 8 & 5 & 8 & 6 & 8 \\
\hline IN9 & 9 & 7 & 6 & 6 & 6 \\
\hline IN10 & 8 & 8 & 8 & 5 & 8 \\
\hline TC & 78 & 75 & 77 & 56 & 76 \\
\hline
\end{tabular}

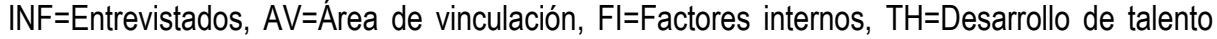
humano, TV=Tipo de vinculación, TC=Trabajo colaborativo, TC=Total categoría, IN= Entrevistado.

Fuente: Elaboración propia

La Tabla 5, recoge la información descriptiva para cada uno de los factores organizacionales, se tiene una muestra de 10 casos, con un promedio de dimensiones (menciones) por los entrevistados de 7.80, 7.50, 7.70, 5.60 y 7.60, así mismo una desviación típica de .632, 1.434, $1.059,1.838$ y .966 para cada factor, y un rango de valores (menciones) que oscilan entre $2 \mathrm{y}$ 10.

Tabla 5. Estadísticos

\begin{tabular}{|ll|r|r|r|r|r|}
\hline & & AV & \multicolumn{1}{c|}{ FI } & \multicolumn{1}{l|}{ TH } & \multicolumn{1}{l|}{ TV } & \multicolumn{1}{c|}{ TC } \\
\hline N & Válidos & 10 & 10 & 10 & 10 & 10 \\
& Perdidos & 0 & 0 & 0 & 0 & 0 \\
Media & & 7,80 & 7,50 & 7,70 & 5,60 & 7,60 \\
Mediana & & 8,00 & 7,50 & 8,00 & 6,00 & 8,00 \\
Moda & & 8 & $7(a)$ & 8 & 6 & 8 \\
Desv. típ. & &, 632 & 1,434 & 1,059 & 1,838 &, 966 \\
Mínimo & & 7 & 5 & 6 & 2 & 6 \\
Máximo & & 9 & 10 & 9 & 8 & 9 \\
Percentiles & 25 & 7,00 & 6,75 & 6,75 & 4,50 & 6,75 \\
& 50 & 8,00 & 7,50 & 8,00 & 6,00 & 8,00 \\
& 75 & 8,00 & 8,25 & 8,25 & 7,00 & 8,00 \\
\hline
\end{tabular}

a Existen varias modas. Se mostrará el menor de los valores.

$\mathrm{AV}=$ Área de vinculación, Fl=Factores internos, TH=Desarrollo de talento Humano, TV=Tipo de vinculación, TC=Trabajo colaborativo.

Fuente: Elaboración propia 
Factores organizacionales que influyen en la generación de vinculación entre empresas y universidades

\section{Gráfica 1. Diagrama de Cajas y bigotes.}

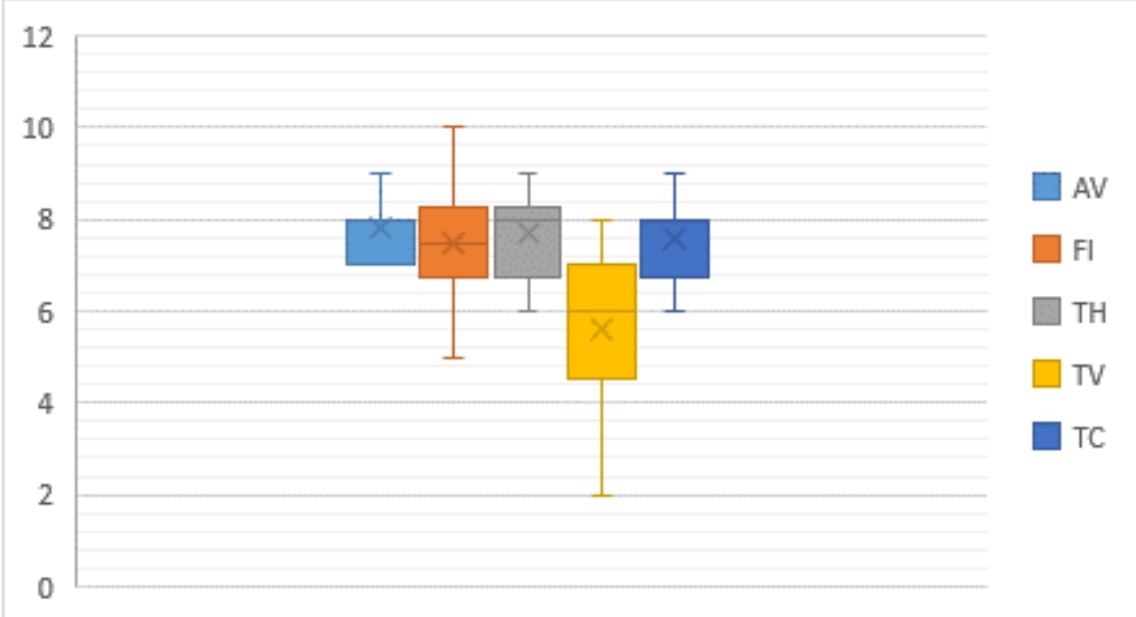

$\mathrm{AV}=$ Área de vinculación, Fl=Factores internos, $\mathrm{TH}=$ Desarrollo de talento humano, TV=Tipo de vinculación, TC=Trabajo colaborativo.

Fuente: Elaboración propia

En el gráfico 1 Cajas y bigotes pueden observarse los siguientes datos para cada factor.

Para el factor AV (Área de vinculación) un cuarto de los valores de los datos se localiza en 7, un cuarto de los valores de los datos se localiza entre 7 y 8 , un cuarto de los valores de los datos se localiza en 8 , y un cuarto de los valores de los datos se localiza entre 8 y 9 .

Así mismo, para el factor $\mathrm{FI}$ (Factores internos) un cuarto de los valores de los datos se localiza entre 5 y 6.75 , un cuarto de los valores de los datos se localiza entre 6.75 y 7.5 , un cuarto de los valores de los datos se localiza entre 7.5 y 8.25 , y un cuarto de los valores de los datos se localiza entre 8.25 y 10.

En cuanto al factor TH (Desarrollo de talento humano) un cuarto de los valores de los datos se localiza entre 6 y 6.75 , un cuarto de los valores de los datos se localiza entre 6.75 y 8 , un cuarto de los valores de los datos se localiza entre 8 y 8.25 , y un cuarto de los valores de los datos se localiza entre 8.25 y 9 .

De igual manera para el factor TV (Tipo de vinculación) un cuarto de los valores de los datos se localiza entre 2 y 4.5 , un cuarto de los valores de los datos se localiza entre 4.5 y 6 , un cuarto de los valores de los datos se localiza entre 6 y 7 , y un cuarto de los valores de los datos se localiza entre 7 y 8.

Por último, para el factor TC (Trabajo colaborativo) un cuarto de los valores de los datos se localiza entre 6 y 6.75 , un cuarto de los valores de los datos se localiza entre 6.75 y 8 , un cuarto de los valores de los datos se localiza en 8 , y un cuarto de los valores de los datos se localiza entre 8 y 9.

Así mismo se realizó la prueba de suma de rangos de Kruskal-Wallis para múltiples muestras independientes y determinar si cualquiera de las diferencias entre las medianas es estadísticamente significativa, para lo cual se comparó el valor $\mathbf{p}$ con el nivel de significancia 
para evaluar la hipótesis nula, en donde la hipótesis nula indica que las medias de población son todas iguales, se tomó un nivel de significancia (denotado como $\alpha$ o alfa) de $\mathbf{0 . 0 5}$. En donde, un nivel de significancia de $\mathbf{0 . 0 5}$ indica un riesgo de $\mathbf{5 \%}$ de concluir que existe una diferencia cuando no hay una diferencia real. El valor $\boldsymbol{p}$ es una probabilidad que mide la evidencia en contra de la hipótesis nula. Las probabilidades más bajas proporcionan una evidencia más fuerte en contra de la hipótesis nula.

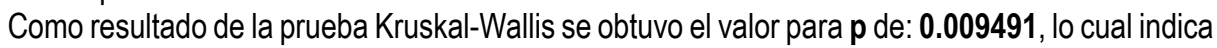
que el valor de $\mathbf{p}$ está en 0 (cero) por debajo del umbral crítico respetable de $\mathbf{0 . 0 5}$, por lo tanto, se rechaza la hipótesis nula debido a que no todas las medianas son iguales. Así mismo, la tabla 6 muestra los resultados de prueba comparativa post hoc Dunn realizada para discernir cuál de los pares tiene diferencias significativas haciendo un ajuste por contrastes múltiples para controlar la probabilidad de cometer algún error en la familia de contrastes considerados (FWER) (Family Wise Error Date) de Holm.

Tabla 6. Valores $p$ de Dunn ajustados por el método Holm FWER.

\begin{tabular}{|c|c|c|c|c|}
\hline & AV & FI & TC & TH \\
\hline FI & 1.000000 & & & \\
\hline TC & 1.000000 & 1.000000 & & \\
\hline TH & 1.000000 & 1.000000 & 1.000000 & \\
\hline TV & 0.019279 & 0.095526 & 0.041413 & 0.023925 \\
\hline
\end{tabular}

$\mathrm{AV}=$ Área de vinculación, Fl=Factores internos, TH=Desarrollo de talento humano, TV=Tipo de vinculación, TC=Trabajo colaborativo.

Fuente: Elaboración propia

Como se puede apreciar en la tabla 6 el grado de significancia del factor TV (Tipo de vinculación) con el factor AV (Área de vinculación) es de $\mathbf{0 . 0 1 9 2 7 9}$, así mismo con el factor TC (Trabajo colaborativo) el grado de significancia es de $\mathbf{0 . 0 4 1 4 1 3}$ y con el factor TH (Desarrollo de talento humano) el grado de significancia es de $\mathbf{0 . 0 2 3 9 2 5}$.

La tabla 7, por último, ofrece la información necesaria para tomar una decisión sobre la hipótesis de bondad de ajusta: el valor del estadístico chi-cuadrado (13.396965), así como el valor de $p$ obtenido de la prueba Kruskal-Wallis (0.009491). Puesto que el crítico es menor que $\mathbf{0 . 0 5}$, se puede rechazar la hipótesis de bondad de ajusta y concluir que las categorías TV (Tipo de vinculación), AV (Área de vinculación), TC (Trabajo colaborativo) y TH (Desarrollo de talento humano) no se ajustan a una distribución uniforme.

Tabla 7. Estadístico Chi-cuadrado

\begin{tabular}{|l|l|}
\hline Chi-cuadrado & 13.396965 \\
\hline Valor $\mathbf{p}$ & 0.009491 \\
\hline
\end{tabular}

Fuente: Elaboración propia

Los resultados obtenidos en el estudio cualitativo, demuestran que para generar la vinculación entre las empresas y universidades es necesario identificar los factores organizacionales, 
Factores organizacionales que influyen en la generación de vinculación entre empresas y universidades

además de trabajar de manera colaborativa y contar con la existencia de un área de vinculación que permita la integración de representantes de empresas y universidades, así como identificar el tipo de vinculación para la generación de proyectos que permitan desarrollar el talento humano. Lo cual, se logró validar con el estudio cuantitativo, demostrando que el factor de mayor significancia sobre los factores de área de vinculación (0.019279), factores internos $(0.095526)$, trabajo colaborativo (0.041413) y desarrollo de talento humano (0.023925), es el factor tipo de vinculación, lo que representa que existe una relación entre los resultados obtenidos del estudio cualitativo y cuantitativo por lo que el objetivo de la investigación se cumplió al identificar los factores organizacionales que influyen en la generación de vinculación entre empresas y universidades.

\section{Conclusiones y discusiones}

Sin duda, la participación de los directivos de las empresas contribuyó a identificar los factores organizacionales que influyen en la generación de vinculación entre empresas y universidades. Esta participación permitió lograr el objetivo de la investigación debido a que se identificaron los factores organizacionales a través del proceso de codificación abierta en donde se desarrollaron las categorías principales y sus dimensiones.

Derivado de la codificación abierta se obtuvieron los factores organizacionales de acuerdo a lo indicado por los entrevistados, siendo estos factores, área de vinculación, factores internos, desarrollo de talento humano, así como el tipo de vinculación y trabajo colaborativo, mismas que permitieron el desarrollo de una propuesta de modelo basado en teoría fundamentada que permita la generación de vinculación entre empresas y universidades.

Así mismo, haciendo uso de los resultados obtenidos de las entrevistas aplicadas a los directivos de empresas se aplicó la prueba de rangos de Kruskal-Wallis, confirmando que el factor de tipo de vinculación influye significativamente con los factores de área de vinculación, trabajo colaborativo y desarrollo de talento humano, por lo que es recomendable que los directivos de las empresas cuenten con el factor de tipo de vinculación bien definido e identificado en su organización, para que el área de vinculación de la empresa tenga conocimiento del tipo de vinculación a desarrollar, y con base a ello, definan las estrategias de trabajar colaborativamente tanto en el interior de la empresa como con otras empresas y universidades, para lo cual es de gran importancia tener conocimiento de las necesidades del mercado, y con base en ello desarrollar el talento humano lo cual permitiría a las empresas y universidades logro de los objetivos en conjunto.

El artículo comprueba que si se aplica la entrevista a una población con características similares es muy probable que se obtengan las mismas significancias. Así mismo, el artículo propone un punto de partida para realizar investigaciones futuras que permitan corroborar su utilidad. La necesidad de investigación futura es clara para dar seguimiento a los hallazgos identificados en el desarrollo de la investigación.

\section{Referencias Bibliográficas}

Alcántar Enríquez, Víctor Manuel y Arcos Vega, José Luis. (2004). La vinculación como instrumento de imagen y posicionamiento de las instituciones de educación superior. Revista Electrónica de Investigación Educativa. Volumen 6, No. 1. México. (Pp. 112). 
Bagella, Michelle y Becchetti Leonardo. (2000). The Competitive Advantage of Industrial Districts: Theoretical and Empirical Analysis. Editorial Physica-Verlag Heidelberg. Italia.

Carbonell, Pilar y Rodríguez-Escudero Ana. (2009). Relationships among team's organizational context, innovation speed, and technological uncertainty: An empirical analysis. Journal of Engineering and Technology Management. Volumen 26. Netherlands (Pp. 28-45).

Chiang Vega, María, Salazar Botello, C., Huerta Rivera, Patricia \& Núñez Partido, Antonio. (2008). Clima organizacional y satisfacción laboral (Instituciones públicas) Desarrollo, adaptación y validación de instrumentos. Revista Universum. Volumen 2, No. 23. Chile (Pp. 66-85).

Díaz Bravo, Laura, Torruco García, Uri, Martínez Hernández, Mildred, \& Varela Ruiz, Margarita. (2013). La entrevista, recurso flexible y dinámico. Investigación en Educación Médica. Volumen 2, No 7. México. (Pp. 162-167).

Kotler Philip y Armstrong Gary. (2001). Fundamentos de marketing. Pearson Education. México.

Lara Rivero, A. (2001). Convergencia tecnológica y nacimiento de las maquiladoras de tercera generación: el caso Delphi-Juárez. Región y Sociedad, Volumen 13, No. 21. México (Pp. 37-77).

Martínez Rizo, Felipe. (1999). Nueve retos para la educación superior. Funciones, actores y estructuras. Extraído

http://www.fmrizo.net/fmrizo pdfs/libros/L\%2025\%202000\%20Nueve\%20retos $\% 20$ pa ra\%20la\%20Educacion\%20Superior\%20ANUIES.pdf consulta: 24/03/19.

Pérez Esparza, Aimee. (2016). Razones que motivan la vinculación de la universidad con la empresa: Análisis comparativo México y Argentina. Revista RAITES (antes Panorama Administrativo). Volumen 2, No. 4. México (Pp. 10-28).

Porter, Michael (1998). Ser competitivo: nuevas aportaciones y conclusiones. Ed. Deusto, España.

Saavedra G., María L. (2009). Problemática y desafíos actuales de la vinculación universidad empresa: El caso mexicano. Actualidad Contable Faces. Volumen 12, No. 19. Venezuela. (Pp. 100-119).

Sarduy Domínguez, Yanetsys. (2007). El análisis de información y las investigaciones cuantitativa y cualitativa. Revista Cubana de Salud Pública. Volumen 33, No. 3. Cuba. (Pp. 1-11).

Strauss, Anselm y Corbin, Juliet. (1990). Basics of Qualitative Research: Grounded Theory, procedures and techniques (fourth edition). Sage Publications. Newbury Park, Estados Unidos.

Velázquez Valadez, Guillermo. (2012). Impacto de la comunicación organizacional, en la competitividad y la vinculación de las empresas mexicanas con la universidad. Recherches en Sciences de Geston.Volumen 3, No. 90. Francia. (Pp. 89-119). 
Factores organizacionales que influyen en la generación de vinculación entre empresas y universidades

Anexo I

\section{Entrevista}

Fenómeno de estudio: Identificar los factores organizacionales que generan la vinculación entre empresas y universidades.

Introducción - Proyecto e Investigador:

Introducción: Hola mi nombre es....

Estoy realizando una investigación para identificar los factores organizacionales que influyen en la generación de la vinculación entre empresa y universidades.

Voy a hacerle algunas preguntas sobre lo que piensa con base en su experiencia acerca de la organización interna de la empresa para generar la vinculación entre empresas y universidades, trabajo colaborativo para la generación de la vinculación, desarrollo de talento humano en la empresa, estrategias de talento humano para disminuir su deserción y aumentar la retención, así como proyectos de vinculación entre empresas y universidades. Durante esta entrevista, no hay respuestas correctas o incorrectas. Sólo me interesa lo que usted piensa. Sé que algunas de estas preguntas pueden parecer muy grandes. Por favor, trate de responder tan honestamente como pueda. Para el estudio, sólo estamos tratando de averiguar lo que usted opina sobre estos temas. Si le parece bien, voy a grabar la entrevista.

\section{Demográfico (Preguntas sobre los entrevistados):}

¿Cuál es su nombre y qué edad tiene?

Nombre de la empresa.

¿Qué cargo o puesto tiene en la empresa?

Preguntas acerca de la organización interna de la empresa para generar la vinculación entre empresas y universidades

1.- ¿Cuáles son los tipos de vinculación que se llevan a cabo?

2.- ¿Cuáles son las razones por las que realizan vinculación?

3.- ¿Cómo se encuentra integrado el departamento o área de vinculación en la empresa?

4.- ¿De qué manera se encuentran formalizados los procesos de vinculación?

5.- ¿Qué factores organizacionales considera son importantes para generar la vinculación con universidades?

6.- ¿Cómo influyen los factores organizacionales en la generación de la vinculación con universidades?

7.- ¿De qué manera se lleva a cabo el seguimiento a la vinculación?

Preguntas acerca del trabajo colaborativo para la generación de la vinculación entre empresas y universidades

8.- ¿Cuáles son las empresas o grupos en los que participa para la generación de vinculación?

9.- ¿Cuáles son las principales barreras a las que se han enfrentado por parte de empresas 0 grupos al momento de proponer trabajar de forma colaborativa?

10.- ¿De qué manera favorece a la empresa trabajar de manera colaborativa con otras empresas?

11.- ¿Qué factores de trabajo colaborativo considera importantes para la generación de vinculación?

12.- ¿Qué espera obtener de las empresas al trabajar de forma colaborativa?

Preguntas acerca del desarrollo del talento humano en la empresa

13.- ¿De qué manera se realiza la gestión de talento humano? 
14.- ¿Cómo logra la fidelización de su talento humano?

15.- ¿De qué manera capacitan al talento humano?

16.- ¿Cuáles son las competencias con las que debe de contar el talento humano?

17.- ¿De qué forma garantiza la eficacia y el máximo desarrollo del talento humano?

Preguntas acerca de las estrategias de talento humano para disminuir su deserción y aumentar la retención

18.- ¿Qué factores considera que se deben de tomar en cuenta para aumentar la retención de talento humano?

19.- ¿Qué factores considera que se deben de tomar en cuenta para disminuir la deserción de talento humano?

Preguntas acerca de los proyectos de vinculación entre empresas y universidades

20.- ¿Cuáles son los proyectos de vinculación en los que ha participado?

21.- ¿Cuáles considera que sean los principales factores organizacionales para realizar proyectos de vinculación?

22.- ¿Qué beneficios ha logrado obtener la empresa derivado de la vinculación con empresas o universidades?

23.- ¿Actualmente cuáles son los proyectos de vinculación que se desarrollan en la empresa con otras empresas o universidades? 